\title{
The initial conditions of high-mass star formation: radiative transfer models of IRDCs seen in the Herschel` Hi-GAL survey
}

\author{
L. A. Wilcock ${ }^{1}$, J. M. Kirk ${ }^{1}$, D. Stamatellos ${ }^{1}$, D. Ward-Thompson ${ }^{1}$, A. Whitworth ${ }^{1}$, C. Battersby ${ }^{2}$, C. Brunt ${ }^{3}$,

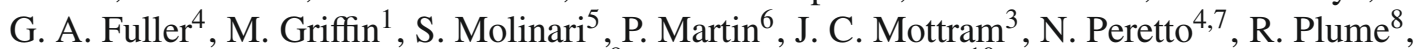 \\ H. A. Smith ${ }^{9}$, and M. A. Thompson ${ }^{10}$ \\ 1 School of Physics and Astronomy, Cardiff University, Queen's Building, Cardiff, CF24 3AA, UK \\ e-mail: lucy.wilcock@astro.cf.ac.uk \\ 2 Center for Astrophysics \& Space Astronomy, University of Colorado, Boulder, CO, 80309, USA \\ 3 School of Physics, University of Exeter, Stocker Road, Exeter, EX4 4QL, UK \\ 4 Jodrell Bank Centre for Astrophysics, School of Physics and Astronomy, University of Manchester, Manchester, M13 9PL, UK \\ 5 Instituto di Fisica dello Spazio Interplanetario, CNR, via Fosso del Cavaliere, 00133 Roma, Italy \\ ${ }^{6}$ Canadian Institute for Theoretical Astrophysics, University of Toronto, Toronto, Canada, M5S 3H8 \\ 7 Laboratoire AIM, CEA/DSM-CNRS-Université Paris Diderot, IFRU/Service d'Astrophysique, C.E. Saclay, Orme des merisiers, \\ 91191 Gif-sur-Yvette, France \\ ${ }^{8}$ University of Calgary, Dept Physics-Astronomy, Calgary, AB T2N 1N4, Canada \\ 9 Harvard-Smithsonian Center for Astrophysics, 60 Garden Street, Cambridge, MA, 02138, USA \\ 10 Centre for Astrophysics Research, Science and Technology Research Institute, University of Hertfordshire, AL10 9AB, UK
}

Recieved 28 July 2010 / Accepted 7 December 2010

\section{ABSTRACT}

\begin{abstract}
The densest infrared dark clouds (IRDCs) may represent the earliest observable stage of high-mass star formation. These clouds are very cold, hence they emit mainly at far-infrared and sub-mm wavelengths. For the first time, Herschel has provided multi-wavelength, spatially resolved observations of cores within IRDCs, which, when combined with radiative transfer modelling, can constrain their properties, such as mass, density profile and dust temperature. We use a 3D, multi-wavelength Monte Carlo radiative transfer code to model in detail the emission from six cores in three typical IRDCs seen in the Hi-GAL survey (G030.50+00.95, G031.03+00.26 and G031.03+00.76), and thereby to determine the properties of these cores and compare them with their low-mass equivalents. We found masses ranging from 90 to $290 M_{\odot}$ with temperatures from 8 to $11 \mathrm{~K}$ at the centre of each core and 18 to $28 \mathrm{~K}$ at the surface. The maximum luminosity of an embedded star within each core was calculated, and we rule out the possibility of significant high mass star formation having yet occurred in three of our cores.
\end{abstract}

Key words. stars: formation - ISM: clouds - dust, extinction

\section{Introduction}

We present observations, performed with the ESA Herschel Space Observatory (Pilbratt et al. 2010), of a section of the Galactic plane. We use Herschel's large collecting area and powerful science payload to perform imaging photometry using the PACS (Photodetector and Array Camera Spectrometer; Poglitsch et al. 2010) and SPIRE (spectral and photometric imaging receiver; Griffin et al. 2010) instruments. These observations were carried out as part of Herschel's Science Demonstration Phase (SDP) for the Herschel Infrared Galactic Plane Survey (Hi-GAL; Molinari et al. 2010a,b) open time Key Project to map the inner Galactic Plane. In particular, we are studying cores within the infrared dark clouds (IRDCs) found in the Galactic Plane.

IRDCs were intially discovered by the ISO (Perault et al. 1996) and MSX (Carey et al. 1998; Egan et al. 1998) surveys as regions of high contrast against the mid-infrared (MIR) background. The densest IRDCs may eventually form massive stars,

\footnotetext{
* Herschel is an ESA space observatory with science instruments provided by European-led Principal Investigator consortia and with important participation from NASA.
}

and are therefore presumed to represent the earliest observable stage of high mass star formation. Some IRDCs contain cold, compact cores, which are believed to be the high mass equivalent of low-mass prestellar cores.

IRDCs and their cores have low temperatures $(T<25 \mathrm{~K}$; e.g. Egan et al. 1998; Teyssier et al. 2002) and therefore emit mostly at far-infrared (FIR) and sub-mm wavelengths. However, until now, spatially resolved observations have not been available in the FIR. Herschel changes this, and offers high resolution FIR images of not only IRDCs, but of the individual cores within - potentially allowing, for the first time, temperatures to be determined and column densities and masses to be accurately constrained for a large number of IRDCs. In this paper we present some first examples of how the Herschel data can be used to trace the physical parameters of cores within IRDCs.

\section{A sample of IRDC cores in the Hi-GAL science demonstration fields}

Hi-GAL is an open time key project of the Herschel Space Observatory and is performing a survey of the inner Galactic Plane $\left(|l|<60^{\circ},|b|<1^{\circ}\right)$ using the PACS and SPIRE instruments. 
Table 1. The physical properties of the six cores.

\begin{tabular}{lllllll}
\hline \hline & \multicolumn{2}{l}{ G030.50+00.95 } & \multicolumn{2}{l}{ G031.03+00.26 } & \multicolumn{2}{c}{ G031.03+00.76 } \\
& Core A & Core B & Core A & Core B & Core A & Core B \\
\hline RA (2000) & $18: 43: 34.7$ & $18: 43: 34.9$ & $18: 47: 01.5$ & $18: 47: 04.2$ & $18: 45: 10.2$ & $18: 45: 17.3$ \\
Dec $(2000)$ & $-01: 42: 29$ & $-01: 44: 36$ & $-01: 34: 36$ & $-01: 33: 39$ & $-01: 20: 00$ & $-01: 19: 12$ \\
Name in PF09 & SDC30.442 & 0.958 & SDC31.039+0.241 & Not included \\
Distance (pc) & 3400 & 3400 & 4900 & 4900 & 3400 & 3400 \\
Semi-major Axis (pc) & 0.6 & 0.7 & 0.45 & 0.55 & 0.4 & 1.0 \\
$R_{0}($ pc) & 0.06 & 0.07 & 0.04 & 0.05 & 0.04 & 0.1 \\
Aspect ratio & 1.93 & 3.16 & 2.46 & 2.22 & 1.70 & 1.65 \\
Position Angle & 50 & 85 & 125 & 340 & 335 & 85 \\
$n_{0}\left(\mathrm{H}_{2}\right)\left(\mathrm{cm}^{-3}\right)$ & $1.4 \times 10^{5}$ & $4.5 \times 10^{4}$ & $3.8 \times 10^{5}$ & $4.2 \times 10^{5}$ & $6.0 \times 10^{5}$ & $2.5 \times 10^{4}$ \\
ISRF $F_{\text {Habing }}$ & 8.5 & 18 & 18 & 81 & 17 & 4.8 \\
$\tau_{\theta=90^{\circ} / \tau_{\theta=0^{\circ}}}$ & 1.93 & 3.16 & 2.46 & 2.22 & 1.59 & 1.65 \\
$F_{500 \mu \mathrm{m}}(\mathrm{Jy})$ & $5.0 \pm 0.5$ & $9.0 \pm 0.9$ & $3.3 \pm 0.3$ & $10 \pm 1$ & $4.5 \pm 0.5$ & $4.4 \pm 0.4$ \\
Model Temperature $(\mathrm{K})$ & $9-19$ & $11-22$ & $9-22$ & $11-28$ & $8-22$ & $10-18$ \\
SED Temperature $(\mathrm{K})$ & $14 \pm 1$ & $16 \pm 2$ & $14 \pm 1$ & $17 \pm 2$ & $14 \pm 1$ & $14 \pm 1$ \\
Model mass $\left(M_{\odot}\right)$ & 120 & 140 & 170 & 290 & 110 & 90 \\
SED mass $-\kappa=0.05 \mathrm{~cm}^{2} \mathrm{~g}^{-1}\left(M_{\odot}\right)$ & 130 & 170 & 170 & 310 & 110 & 100 \\
SED mass $-\kappa=0.03 \mathrm{~cm}^{2} \mathrm{~g}^{-1}\left(M_{\odot}\right)$ & 210 & 280 & 280 & 510 & 180 & 160 \\
$\ln \left(\frac{I_{1}}{I_{2}}\right)$ & 0.75 & - & 1.50 & - & 1.25 & - \\
$L_{\text {max }}\left(L_{\odot}\right)$ & $<6$ & $<3$ & $<200$ & $<1000$ & $<40$ & $<9$ \\
\hline
\end{tabular}

Notes. $R_{0}$ is the flattening radius within the core, $n_{0}\left(\mathrm{H}_{2}\right)$ is the central density of each core, ISRF $\mathrm{Habing}_{\text {is }}$ the ISRF of the core at FUV given in multiples of the Habing flux - i.e. $1.6 \times 10^{-3} \mathrm{erg} \mathrm{s}^{-1} \mathrm{~cm}^{-2}$ (Habing 1968), $\tau_{\theta=90^{\circ}} / \tau_{\theta=0^{\circ}}$ is the ratio of optical depths at $\theta=90^{\circ}$ and at $\theta=0^{\circ}$ and $F_{500 \mu \mathrm{m}}$ is the flux density of each core at $500 \mu \mathrm{m}$. The model temperature is the range of temperatures in the core calculated from the model, and the SED temperature was calculated from single-temperature greybody fitting. The model mass was calculated using $\kappa=0.05 \mathrm{~cm}^{2} \mathrm{~g}^{-1}$ at $500 \mu \mathrm{m}$ (Ossenkopf \& Henning 1994). The two SED masses were calculated using the method of Hildebrand (1983), the SED temperature and the flux density at $500 \mu \mathrm{m}$ (see Sect. 4.1). The difference in ISRFs, $\ln \left(\frac{I_{1}}{I_{2}}\right)$, was calculated from the model (see Sect. 4.3). $L_{\text {max }}$ is the maximum luminosity of a star that could be embedded in each core (see Sect. 4.4).

The two are used in parallel mode to map the Milky Way Galaxy simultaneously at five wavelengths, from 70 to $500 \mu \mathrm{m}$, with resolutions up to $5^{\prime \prime}$ at $70 \mu \mathrm{m}$.

Two regions of the Hi-GAL survey were completed during SDP time. The regions are each four degrees square and center around $l=30^{\circ}, b=0^{\circ}$ and $l=59^{\circ}, b=0^{\circ}$. Using these observations and the Simon et al. (2006a, hereafter S06) and Peretto \& Fuller (2009, hereafter PF09) catalogues, IRDCs can be identified in the Galactic plane with none of the uncertainty that exists in using MIR observations alone (e.g. being unable to differentiate between an IRDC and a dip in the MIR background).

PACS data reduction was performed using the Herschel interactive pipeline environment (HIPE; Ott 2010), albeit with some departures from the standard processing (Poglitsch et al. 2010). Most notably, the standard deglitching and crosstalk correction were not used due to poor results in these fields, and custom procedures were written for drift removal.

SPIRE data processing required less deviation from the standard processing methods (Griffin et al. 2010), with both standard deglitching and drift removal producing acceptable results. In both cases, the ROMAGAL generalised least squares algorithm (Molinari et al. 2010a) was used to produce the final maps. A more thorough discussion of the entire data reduction process can be found in Molinari et al. (2010a).

Each of the S06 sources in these regions was studied at 6 wavebands, i.e. Spitzer GLIMPSE $8 \mu \mathrm{m}$ (Benjamin et al. 2003; Werner et al. 2004) and the five Hi-GAL wavelengths (70, 160, $250,350$ and $500 \mu \mathrm{m})$. If the source showed extinction at MIR but emission in the FIR it was identified as an IRDC. Within the $l=30^{\circ}$ region, approximately 330 (PF09) IRDCs were found, each containing at least one infrared dark core.

Our aim is to model those cores without embedded protostars. We focus on three objects, G030.50+00.95, G031.03+00.26 and G031.03+00.76, which meet the criteria for
IRDCs. All three contain two cores each, designated "Core A" and "Core B" in order of right ascension within each cloud. Positions and physical properties of all six cores can be found in Table 1, and images are displayed in Figs. 1-3.

\section{1. $G 030.50+00.95$}

In this cloud, Core B is the more extended and brighter of the two cores and located to the south of Core A (see Fig. 1). In the $250 \mu \mathrm{m}$ and $350 \mu \mathrm{m}$ wavebands, it is possible to see that Core B can be separated into two cores. However, as the individual peaks cannot be seen at either $160 \mu \mathrm{m}$ or $500 \mu \mathrm{m}$, the two are grouped together and modelled as a single core. A distance of $3.4 \mathrm{kpc}$ for the entire cloud is assumed (Simon et al. 2006b). No previously calculated masses are available for these cores. Results calculated from our data are presented in Table 1.

\section{2. $G 031.03+00.26$}

S06 find three cores in this cloud. However, only two can be seen as significant emission sources in the FIR and so here the third is ignored. Core B is the more extended and brighter of the two and is located to the north of Core A (see Fig. 2). Emission from Core B can be clearly seen at wavelengths as short as $160 \mu \mathrm{m}$, whereas emission from Core A is not seen shortward of $250 \mu \mathrm{m}$.

We take a distance of $4.9 \mathrm{kpc}$ for both cores (Teyssier et al. 2002) although we note that there are two emission line components among the line of sight, with the second emission line giving a distance of $6.6 \mathrm{kpc}$. Therefore there is some uncertainty in this distance. Parsons et al. (2009) use $850 \mu \mathrm{m}$ data and find masses of 420 and $560 M_{\odot}$, respectively, for these cores. However, these authors used a different size of aperture. We 
L. A. Wilcock et al.: A radiative transfer model of IRDCs in the Hi-GAL survey
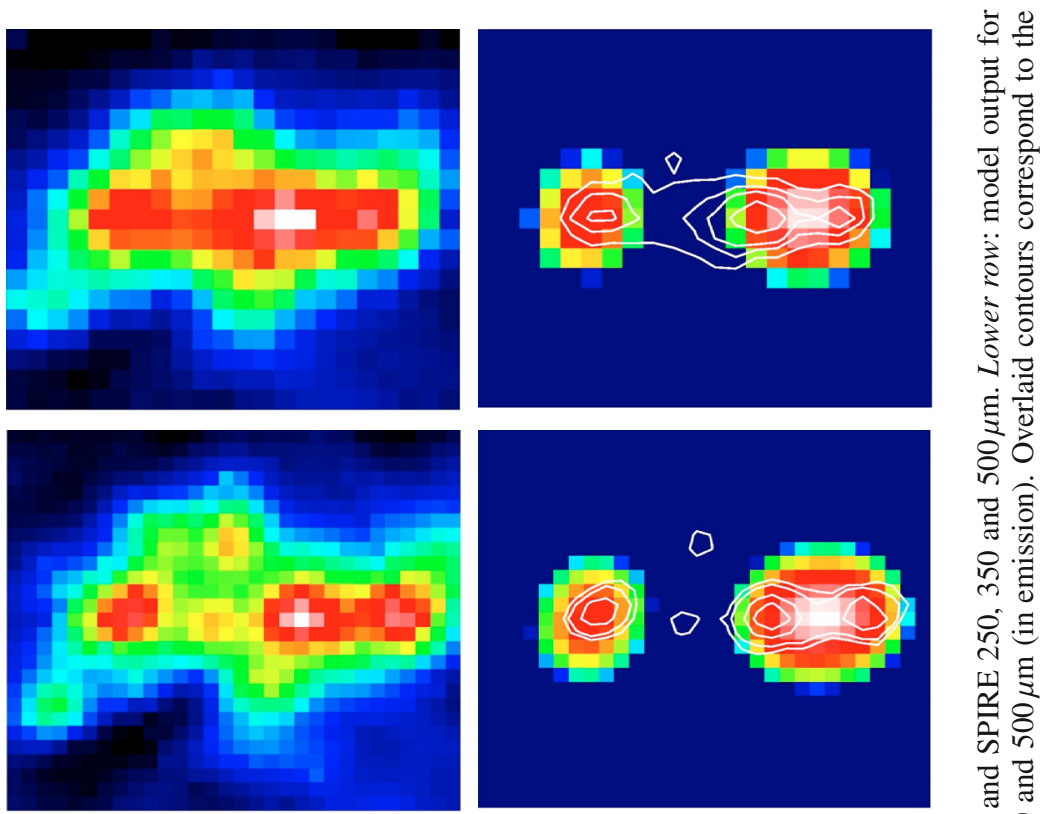

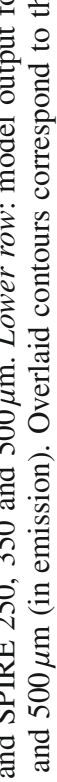
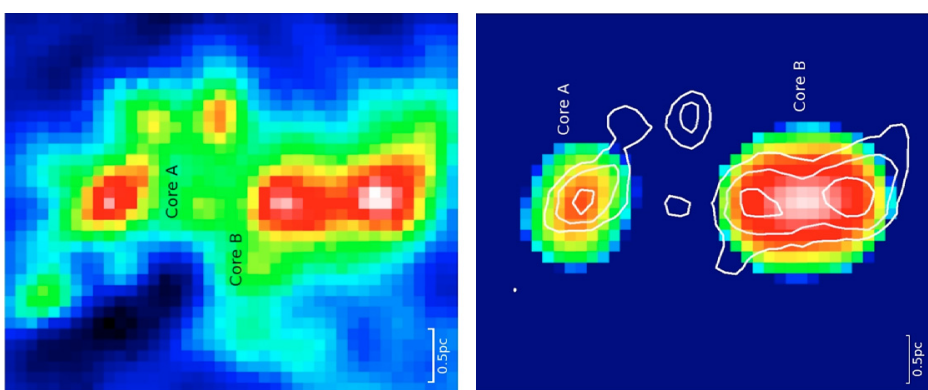

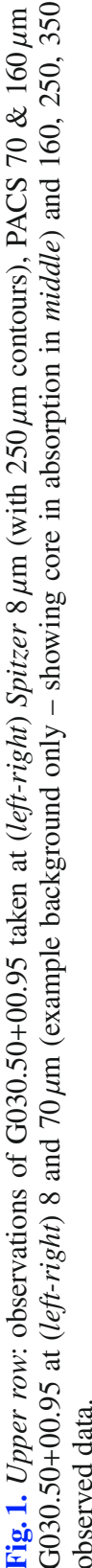
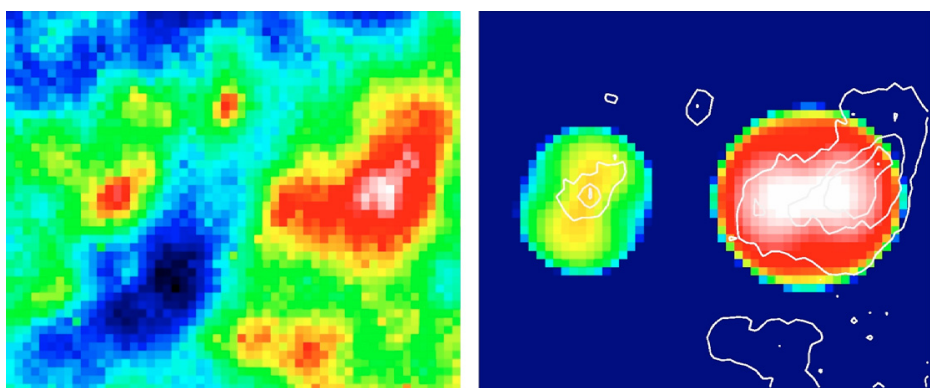

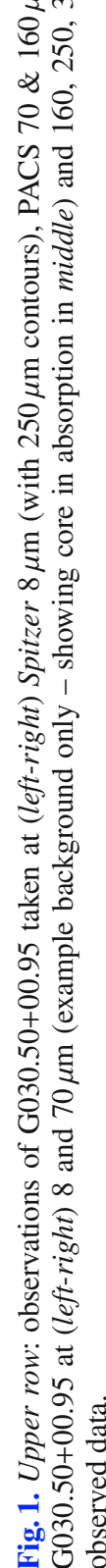
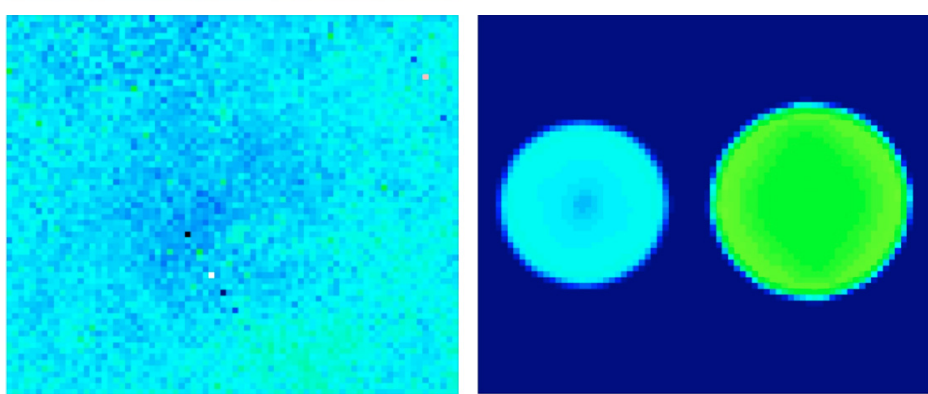

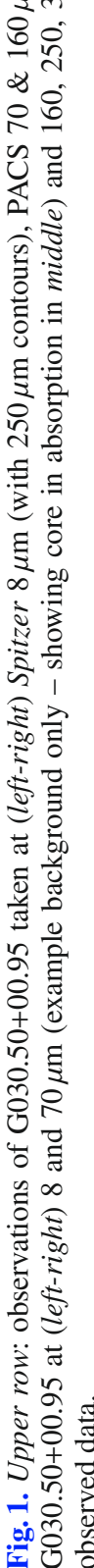
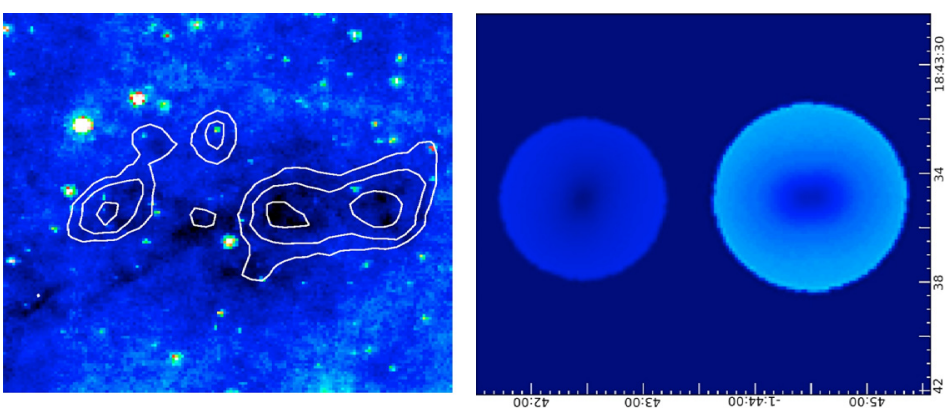
A\&A 526, A159 (2011)
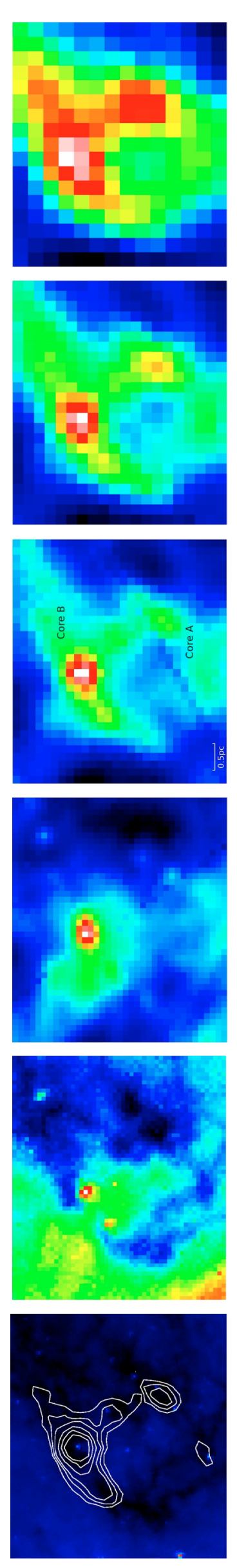
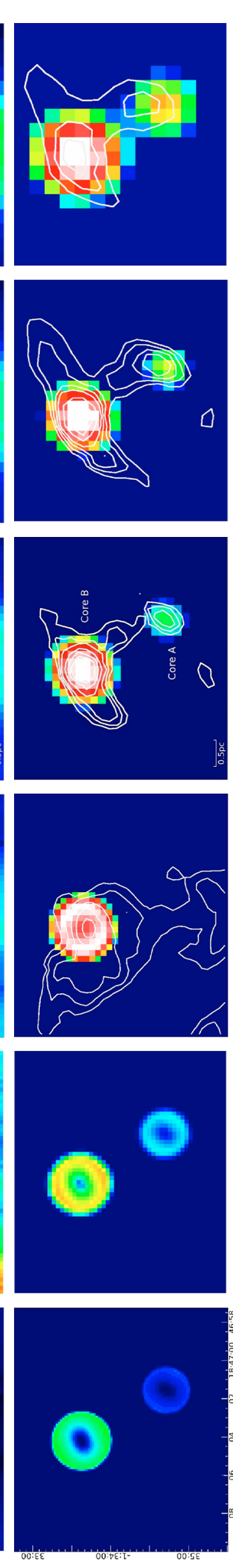
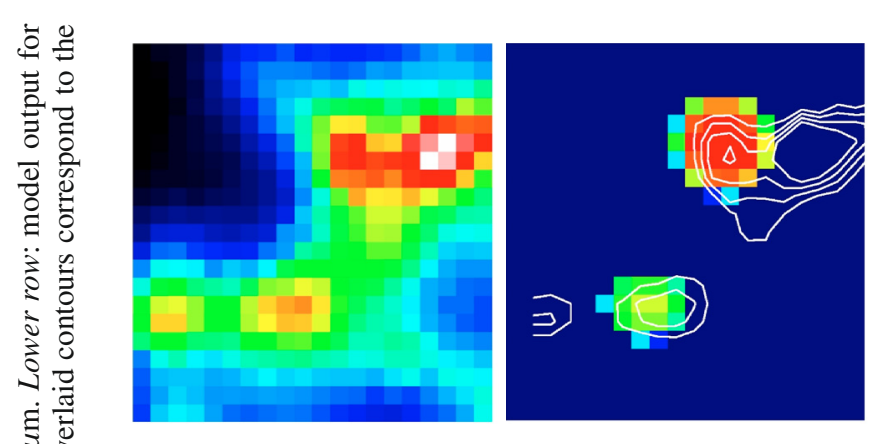

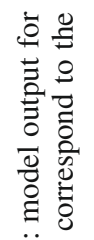
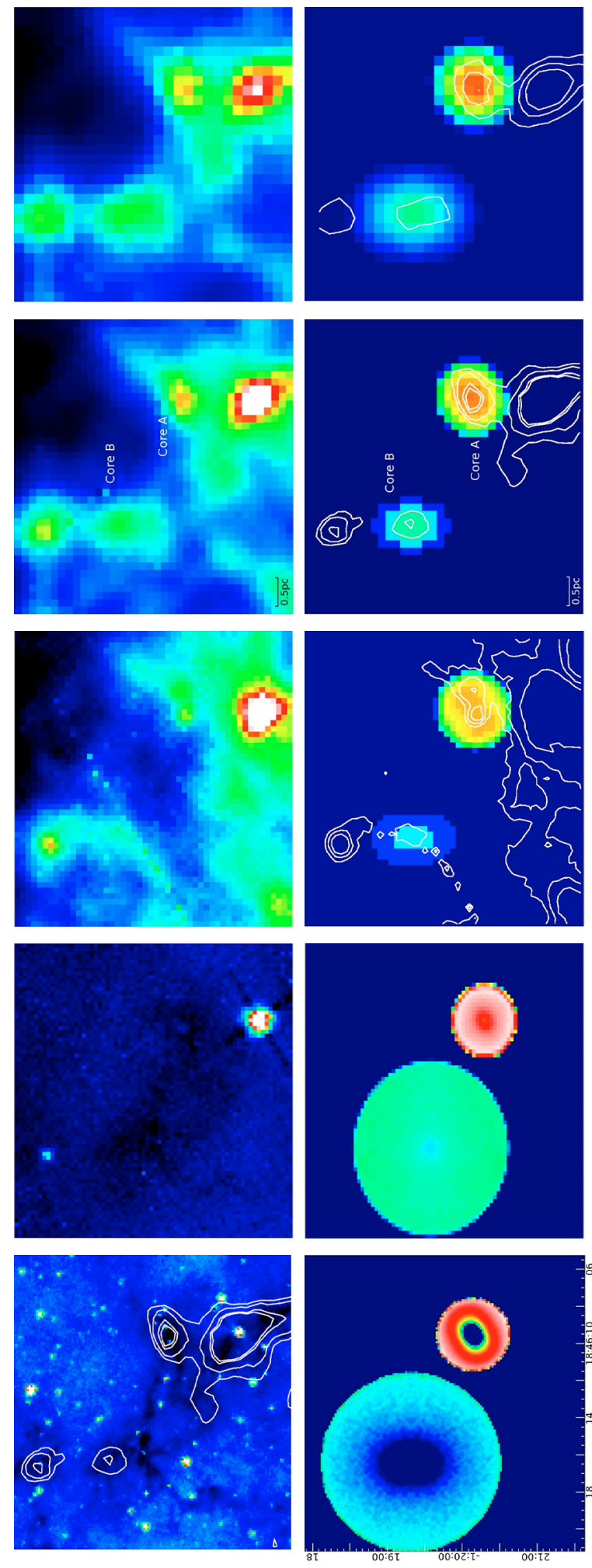

ํํㅇ

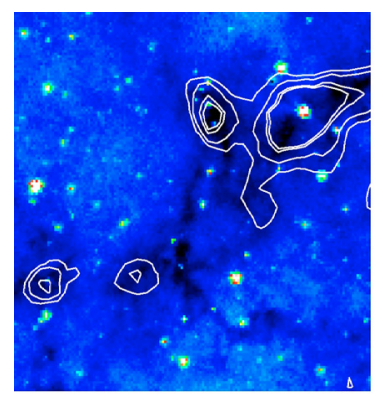

ㅎํำ

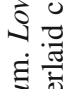

80

로음

员.

ถึํำ

떰홍

完员

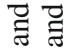

业品

:

สี

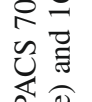

高音

I

:

इ용

责.

远

\$. .0

矛范

का

할

氙

武

을

+

放

प릉

is $\infty$

気

竞

$\ddot{3}$

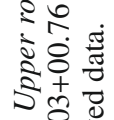

ल.

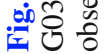


Table 2. The observed, integrated flux densities of the six cores.

\begin{tabular}{lllllll}
\hline \hline Wavelength & \multicolumn{7}{c}{ Flux density (Jy) } \\
& \multicolumn{2}{l}{ G030.50+00.95 } & \multicolumn{2}{l}{ G031.03+00.26 } & \multicolumn{2}{l}{ G031.03+00.76 } \\
& Core A & Core B & Core A & Core B & Core A & Core B \\
\hline $70 \mu \mathrm{m}$ & $<0.03$ & $<3.6$ & $<0.5$ & $<10.1$ & $<1.0$ & $<1.3$ \\
$160 \mu \mathrm{m}$ & 13.4 & 41.9 & 10.9 & 60.3 & 13.7 & 15.4 \\
$250 \mu \mathrm{m}$ & 18.0 & 31.4 & 11.7 & 47.5 & 13.4 & 13.3 \\
$350 \mu \mathrm{m}$ & 9.6 & 16.0 & 6.8 & 23.0 & 8.8 & 8.8 \\
$500 \mu \mathrm{m}$ & 5.0 & 9.0 & 3.2 & 9.7 & 4.5 & 4.4 \\
\hline
\end{tabular}

make a direct comparison with these results in Sect. 4.1 below. Results calculated from our data are presented in Table 1.

\section{3. $\mathrm{G} 031.03+00.76$}

Here, Core B is located to the north of Core A (Fig. 3) and, although it is the more extended of the two, it is actually fainter than Core A. Neither core can be seen in emission at wavelengths shorter than $160 \mu \mathrm{m}$. A distance of $3.4 \mathrm{kpc}$ is assumed (Simon et al. 2006b). No previously calculated masses are available for these cores.

A third core in this cloud, located to the south of Core A, is not modelled here due to a bright $8 \mu \mathrm{m}$ source at the same position, implying the core may have an internal heating source. A possible fourth core exists to the north of Core B, we ignore this as S06 state that it belongs to a different IRDC with no known distance. Results calculated from our data are presented in Table 1.

\section{IRDC models}

We model the cores in two different ways: first, using a greybody single-temperature fit, and second, using the 3D Monte Carlo radiative transfer code, PHAETHON (Stamatellos \& Whitworth 2003, 2005; Stamatellos et al. 2010).

\subsection{Single temperature fitting of SEDs}

The flux density, integrated over twice the FWHM of each core using an elliptical aperture, was measured in all five FIR maps (see Table 2) and an SED (spectral energy distribution) was plotted. These flux densities have been background subtracted, where the background was defined using an off-cloud, elliptical aperture. For example, with G031.03+00.76 the area to the right of Core B was used. The background subtraction removed up to $50 \%$ of the original flux, the exception being at $70 \mu \mathrm{m}$ where over $90 \%$ of the original flux was removed. A single-temperature greybody (modelling thermal emission from cold dust) was fitted (using MPFit; Markwardt 2008) to each core, shown as a dashed line in Fig. 4. This has the form

$F_{v}=B_{v}(T) \Omega\left[\frac{2 m_{\mathrm{H}}}{X} N\left(\mathrm{H}_{2}\right) \kappa_{v}\right]$,

where: $F_{v}$ is the flux density at frequency $v ; B_{v}(T)$ is the blackbody function at temperature $T ; \Omega$ is the solid angle subtended at the observer by the source; $2 m_{\mathrm{H}}$ is the mass of a hydrogen molecule; $X$ is the mass fraction of hydrogen and $\kappa_{v}$ is the dust mass opacity (e.g. Kirk et al. 2010; Ward-Thompson et al. 2010). $\kappa_{v}$ is given by

$\kappa_{v}=0.1 \mathrm{~cm}^{-2} \mathrm{~g}^{-1} \times(v / 1000 \mathrm{GHz})^{\beta}$,

where $\beta$, the dust emissivity index, was set to 1.85 (Ossenkopf \& Henning 1994).
The model was fitted between $160 \mu \mathrm{m}$ and $500 \mu \mathrm{m}$, with the flux density at $70 \mu \mathrm{m}$ being used as an upper limit, as the cores are not visible at this wavelength. The temperature was allowed to vary over a range $3-40 \mathrm{~K}$. The best fit temperatures for the cores are given in Table 1.

\subsection{Radiative transfer modelling of the cores}

The cores were modelled using PHAETHON, a 3D Monte Carlo radiative transfer code. The code uses luminosity packets to represent the ambient radiation field in the system. These packets are injected into the system where they interact (are absorbed, re-emitted or scattered) with it stochastically. The ambient radiation field is taken to be a multiple of a modified version of the Black (1994) interstellar radiation field, which gives a good approximation to the radiation field in the solar neighbourhood.

The input variables of the code are the strength of the ambient radiation field, the density profile, the size and geometry of the core (i.e. spherical, flattened or cometary) and the dust properties of the system. The code calculates the temperature profile of the system as well as SEDs and intensity maps, at different wavelengths and viewing angles.

All six cores showed some measure of eccentricity in the observations and so were modelled with a flattened geometry see Stamatellos et al. $(2004,2010)$ for details. In this case the density profile is given by:

$n(r, \theta)=n_{0}\left(\mathrm{H}_{2}\right) \frac{1+A\left(\frac{r}{R_{0}}\right)^{2}[\sin (\theta)]^{p}}{\left[1+\left(\frac{r}{R_{0}}\right)^{2}\right]^{2}}$,

where $r$ is the radial distance, $\theta$ is the polar angle and $R_{0}$ is the flattening radius (i.e. the radial distance for which the central density is approximately constant). $n_{0}\left(\mathrm{H}_{2}\right)$ is the central density, which is controlled as an input variable. $A$ is a factor that controls the equatorial to polar optical depth ratio and determines how flattened the core is. $p$ determines how quickly the optical depth changes from equator to pole, and was set to 2 .

The FWHM of the major axis of each observed core was measured at $250 \mu \mathrm{m}$ and used as the model core's semi-major axis. The flattening radius, $R_{0}$, was set at one tenth of this value. The central density, $n_{0}\left(\mathrm{H}_{2}\right)$, and interstellar radiation field (ISRF) incident on the core were then varied until the output model's SED matched the observed data. The model SED is shown as a dotted line in Fig. 4. The final values for $n_{0}\left(\mathrm{H}_{2}\right)$ and the incident ISRF are noted in Table 1.

It should be noted that the internal structure of cores within infrared dark clouds have not yet been observed in detail. Hence our assumption of an elliptical geometry may be an oversimplification. If there is structure on smaller scales than we can resolve this might affect our results. For example, small scale fragmentation might allow the ISRF to penetrate further into the cores, meaning that our calculated values are probably upper values. 

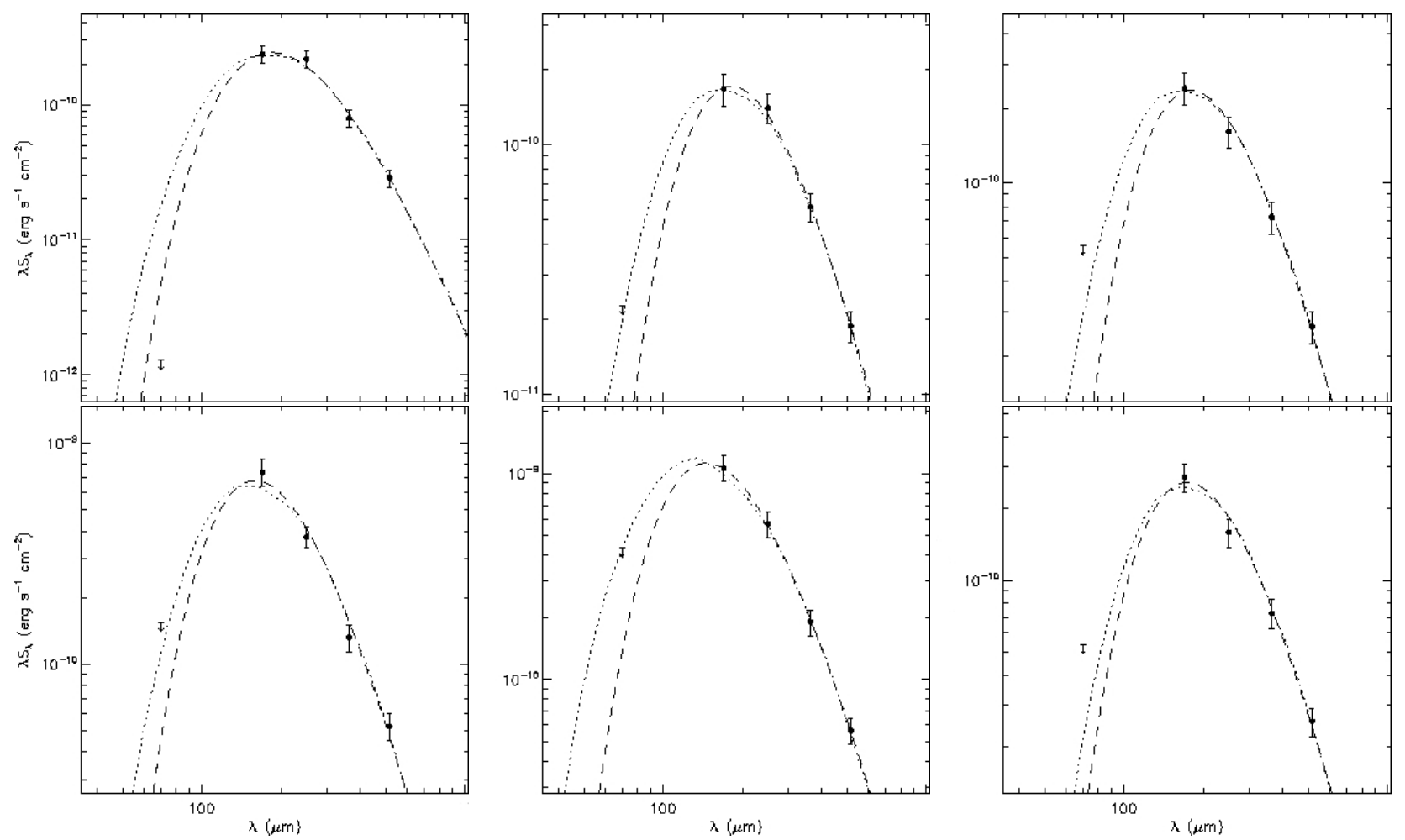

Fig. 4. SEDs of all six cores. Upper row (left-right): G030.50+00.95, G031.03+00.26 and G031.03+00.76 Core A. Lower row (left-right): G030.50+00.95, G031.03+00.26 and G031.03+00.76 Core B. The dotted line shows the SED calculated from PHAETHON, using $\beta=1.85$. The dashed line shows the single-temperature grey-body, with temperatures as noted in Table 1 , also using $\beta=1.85$.

Figures 1-3 show the output of the model at wavelengths corresponding to the wavelengths of the observed data. The modelled images have pixels of $0.02 \times 0.02 \mathrm{pc}$ in size, which corresponds to $8^{\prime \prime}$ pixels for $\mathrm{G} 031.03+00.26$ and $14^{\prime \prime}$ pixels for G030.50+00.95 and G031.03+00.76. All the images have been convolved with the telescope beam. For wavelengths where no emission is visible in the model, an image showing background radiation is shown. As no attempt was made to correctly model the surrounding area, these images are not an accurate representation of the parent cloud but do show the cores in absorption as seen in the observations.

With the possible exception of G31.03+00.26 Core B (which may have evidence of an $8 \mu \mathrm{m}$ point source), the $8 \mu \mathrm{m}$ images shows no emission in either the model or the observed data, as IRDCs do not emit significantly in the MIR due to their low temperatures. The modelled images show emission starting at $250 \mu \mathrm{m}$ for G031.03+00.26 Core A and $160 \mu \mathrm{m}$ for the remaining five cores (albeit only faintly for the cores in G031.03+00.76) and absorption at shorter wavelengths. The modelled emission fits well with the observations, at all wavelengths.

\section{Discussion}

\subsection{Core masses}

The masses of the cores were calculated in two ways: (i) using the radiative transfer models; and (ii) using the observed flux densities at $500 \mu \mathrm{m}$. The latter was carried out using the equation

$M=\frac{F_{v} D^{2}}{B_{v}(T) \kappa_{v}}$,

(Hildebrand 1983), where $D$ is the source distance. We call the former the model mass and the latter the SED mass.
Two different values of opacity at $500 \mu \mathrm{m}$ were used: $\kappa=$ $0.03 \mathrm{~cm}^{2} \mathrm{~g}^{-1}$ (Ossenkopf \& Henning 1994) and $\kappa=0.05 \mathrm{~cm}^{2} \mathrm{~g}^{-1}$ (Preibisch et al. 1993; Henning et al. 1995). The latter is the value used to calculate the masses of low-mass prestellar cores (e.g. Kirk et al. 2005). The model masses were found using the Ossenkopf \& Henning (1994) opacities.

The model masses match well with the SED masses calculated using $K=0.05 \mathrm{~cm}^{2} \mathrm{~g}^{-1}$, but not with those calculated using $\kappa=0.03 \mathrm{~cm}^{2} \mathrm{~g}^{-1}$. Both sets of SED masses and the model masses are shown in Table 1.

The masses calculated here are in agreement with those found in previous studies of IRDCs (e.g. Williams et al. 2001; Simon et al. 2006b; Rathborne et al. 2006). In particular, in Rathborne et al. (2006), masses of dark cores were calculated and found to vary from 10 to $2100 M_{\odot}$. All of our cores fit within this range.

$850 \mu \mathrm{m}$ masses for both cores in $\mathrm{G} 031.03+00.26$ were calculated by Parsons et al. (2009). Core A was found to have a mass of $460 M_{\odot}$ and Core B of $520 M_{\odot}$. When using the same core boundaries as in Parsons et al. (2009) and $k=0.05 \mathrm{~cm}^{2} \mathrm{~g}^{-1}$, we find $500 \mu \mathrm{m}$ masses of $470 M_{\odot}$ and $540 M_{\odot}$, similar to the $850 \mu \mathrm{m}$ masses. This gives us additional confidence in our calculations.

\subsection{Core temperatures}

The energy deposited in the gas due to cosmic ray ionisation heating is much lower than the energy absorbed by the dust due to external heating from the ISRF (Evans et al. 2001). Thus, we can safely assume that the dust temperature is not affected by the energy exchange between gas and dust. The lack of MIR point sources also implies that heating via compression is minimal (Battersby et al. 2010). These cores (again with the possible exception of G031.03+00.26 Core B) therefore have no significant 

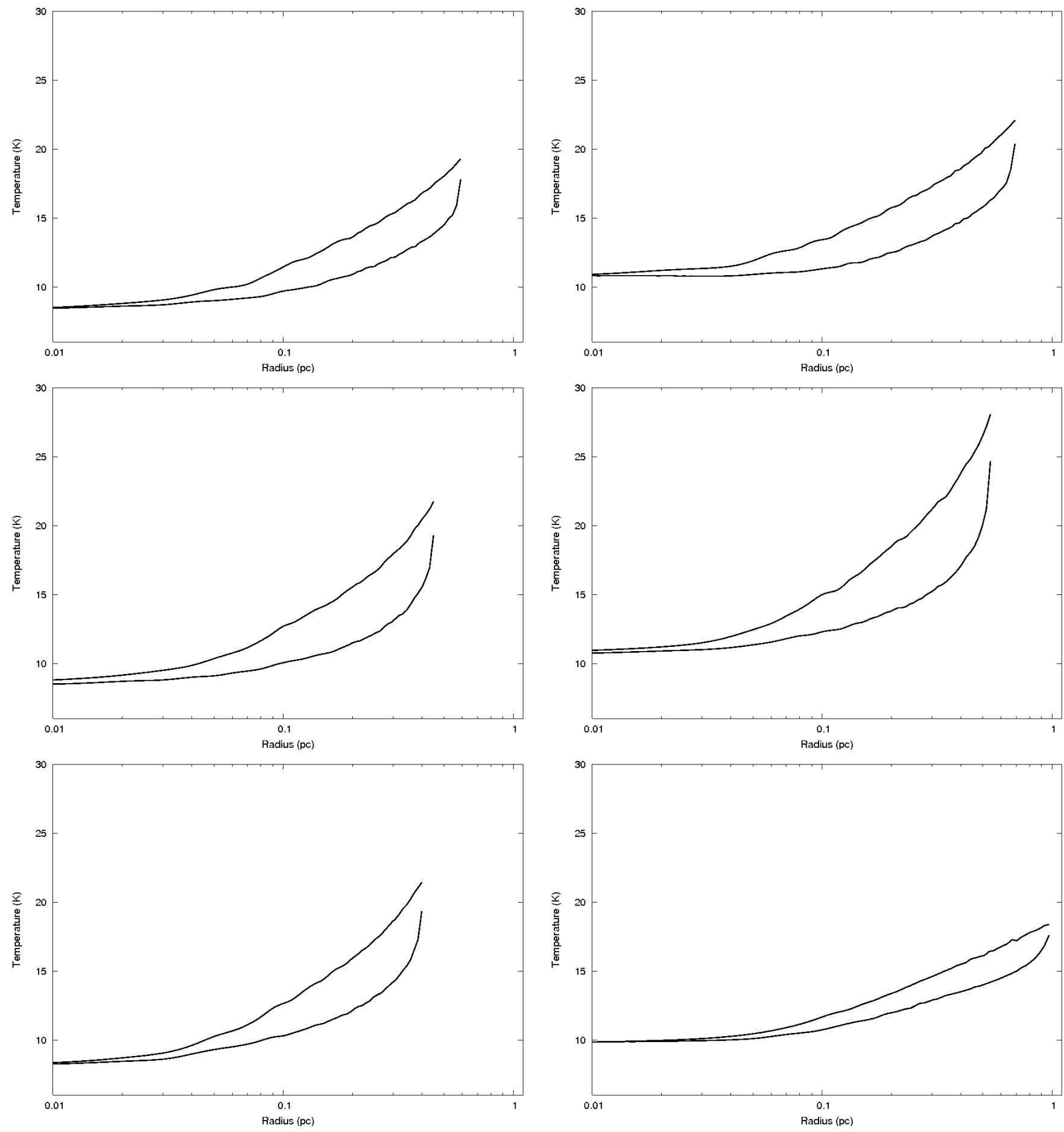

Fig. 5. Dust temperature profiles of the cores at two different directions of the cloud, as calculated from the radiative transfer model. The lower line corresponds to the midplane of the flattened structure $\left(\theta=90^{\circ}\right)$, whereas the upper line corresponds to the direction perpendicular to the midplane $\left(\theta=0^{\circ}\right)$. Upper row: $\mathrm{G} 030.50+00.95$ Cores A and $\mathrm{B}$; middle row: $\mathrm{G} 31.03+00.26$ Cores $\mathrm{A}$ and $\mathrm{B}$; lower row: $\mathrm{G} 31.03+00.76 \mathrm{Cores} \mathrm{A}$ and $\mathrm{B}$.

internal heating sources and are heated mainly by the external radiation field.

The temperature profiles of the modelled cores are shown in Fig. 5. They show a low temperature throughout the centre of the core, before it eventually begins rising, peaking at the outer edges. A higher temperature at the edges than at the centre is expected due to the lack of any internal heating source. Some stepping can be seen in the graphs, this is due to the temperature table having a spacing of $0.2 \mathrm{~K}$.
This gradient is consistent with previous studies of IRDCs (e.g. Peretto et al. 2010) and is similar to what is seen in lowmass prestellar cores (e.g. Nutter et al. 2008). However, IRDCs have higher outer temperatures than low-mass prestellar cores, which can be explained by a higher ISRF around these IRDCs relative to nearby regions of star formation.

As all six cores have a flattened geometry, the temperatureradius profile varies with the direction inside the core. The two extreme cases, $\theta=0^{\circ}$ and $\theta=90^{\circ}$, are shown in the temperatureradius profiles. $\theta=90^{\circ}$ is along the midplane of the structure, 
while $\theta=0^{\circ}$ corresponds to the short axis of a flattened core, perpendicular to the midplane.

The average temperature from the model of each core agrees with the result from the single-temperature greybody fit. However, the temperature-radius profile shows this fit to be an over-simplification, with temperatures within each core varying by $\sim 10 \mathrm{~K}$ and being dependent upon angular direction in the core.

\subsection{The interstellar radiation field}

Five of the six cores required a higher ISRF than the solar neighbourhood value in order to correctly match the observed flux densities at shorter wavelengths (i.e. 160 and $250 \mu \mathrm{m}$ ). As an example we show the cores' ISRF at far-ultraviolet (FUV) in Table 1. This is merely to indicate how the ISRF varies from core to core. For comparison, the solar neighbourhood ISRF at FUV is $7.6 \times 10^{-3} \mathrm{erg} \mathrm{s}^{-1} \mathrm{~cm}^{-2}$ (Black 1994) or 4.6 times the Habing flux $\left(1.6 \times 10^{-3} \mathrm{erg} \mathrm{s}^{-1} \mathrm{~cm}^{-2}\right.$, Habing 1968). The IRDCs typically have an ISRF around four times greater, consistent with them being in the stronger interstellar radiation field of the inner Galactic plane.

Differences between the ISRF required for cores within the same field can be explained by one core being more deeply buried within the parent cloud and thus having less external radiation heating it. The difference between the ISRFs of the cores are listed in Table 1 as $\ln \left(\frac{I_{1}}{I_{2}}\right)$, where $I_{1}$ corresponds to the core that is modelled using a higher ISRF and $I_{2}$ to the core modelled with the lower ISRF (within the same field). This ratio can be thought as a measure of how more deeply a core is embedded when compared with the other core in the same field.

Core B in G031.03+00.26 appears to reside in an unusually intense radiation field, 17 times that of the solar neighbourhood and far higher than any of the other cores, possibly pointing to an abnormally high ISRF in this field. Core A in the same cloud has an ISRF value comparable to the other cores, which implies that it is either more deeply buried within the IRDC than Core B or there is a highly asymmetrical external radiation field incident on this cloud. Alternatively, differences in emission from the cores could be the result of different physical properties (e.g. dust grain size) between different cores in the same IRDC. Core B may not, in fact, be completely externally heated. This one is unusual in our sample, in that it may have an embedded protostar. There is some evidence for an $8 \mu \mathrm{m}$ source associated with this core.

\subsection{Internal heating sources}

A lack of significant internal heating in the modelled cores was assumed due to the absence of any near-infrared or MIR emission. This is further supported by the temperatures of the cores decreasing towards their centres. However, more detailed study into the heating of infrared dark cores needs to be performed before this can be conclusively proven. For now we can put limits on the luminosity of any embedded source.

Using the column-density estimated for each core, we can calculate the visual extinction, the $K$-band extinction, and finally the extinction at $8 \mu \mathrm{m}\left(A_{8}\right.$; Indebetouw et al. 2005). A peak $8 \mu \mathrm{m}$ flux was measured for each core $\left(F_{8}\right)$ and, using

$F=F_{8} 10^{A_{8} / 2.5}$,

the maximum flux density of any embedded source $(F)$ was calculated. This was then converted to a luminosity $(L)$ by multiplying by the Spitzer $8 \mu \mathrm{m}$ bandwidth to convert from flux density to flux and using

$L=4 \pi D^{2} F$.

These values are listed in Table 1 . We note that G31.03+00.76 Core A has some $8 \mu \mathrm{m}$ sources around it's edge, but they do not appear to be centred on the core.

Our upper limits for G30.50+00.95 Cores A and B and G31.03+00.76 Core B are fairly low. We can thus rule out any significant massive star formation having occurred in their centres, making it possible that these cores are the high-mass equivalents of low-mass prestellar cores. For both cores in G031.03+00.26 and in G31.03+00.76 Core A, the upper limits are too high to reach this conclusion.

In the case of G031.03+00.26 Core B a very faint source can be seen at $8 \mu \mathrm{m}$ from which $F_{8}$ was measured. These calculations make the assumption that this source is deeply embedded within the cloud. Should the source instead be in the foreground or towards the nearer edge of the IRDC then the maximum embedded luminosity would be very much lower.

\section{Summary and conclusions}

We have confirmed the status of G030.50+00.95, G031.03+00.26 and G031.03+00.76 as IRDCs using midand far-infrared observations. Six infrared dark cores have been found in the FIR in these IRDCs and modelled using the 3D Monte Carlo radiative transfer code PHAETHON. The radiative transfer models reproduce the observed SEDs and the FIR images of these cores.

The PHAETHON code calculated masses for the cores ranging from 90 to $290 M_{\odot}$. The masses calculated from the SED temperature ranged from $100 M_{\odot}$ to $310 M_{\odot}\left(\right.$ with $\kappa=0.05 \mathrm{~cm}^{2} \mathrm{~g}^{-1}$ ). These are in agreement with previous masses found for infrared dark cores. It should be noted that objects of this size and mass are not likely to create a single star, but rather a cluster of stars.

The greybody fitted SED temperatures varied from 14 to $17 \mathrm{~K}$. The average temperatures calculated from the model agreed with these values. However, the model showed that the single-temperature fit was an over-simplification of the temperatures inside the cores as temperature-radius profiles showed changes in temperature of $\sim 10$ to $17 \mathrm{~K}$ within a single core. The model calculated temperatures of 8 to $11 \mathrm{~K}$ at the centre of each core and 18 to $28 \mathrm{~K}$ at the surface.

Significant temperature gradients were also seen by Peretto et al. (2010), where temperature maps of 22 IRDCs were created and non-uniform temperatures ranging from 10 to $22 \mathrm{~K}$ were found. Stamatellos et al. (2010) used PHAETHON to model an IRDC core and found temperatures from 10 to $21 \mathrm{~K}$, consistent with our results.

The maximum luminosity of a star embedded within each core was found. The upper luminosity limits for three of the cores (G30.50+00.95 Cores A and B and G31.03+00.76 Core B) are low, ruling out the possibility of any significant high mass star formation having yet occured and making it likely that these cores are the high-mass equivalent of prestellar cores.

In most cases, the amount of radiation incident on the cores was found to be higher than that in the local neighbourhood, as expected for objects within active star-forming regions in the Galactic Plane. Differences in the amount of radiation falling on cores in the same cloud were attributed to one core being more deeply buried within the IRDC than the other. This method can now be used on all cores within IRDCs found in the Hi-GAL survey. 
Acknowledgements. L.A.W. acknowledges STFC studentship funding. SPIRE has been developed by a consortium of institutes led by Cardiff University (UK) and including Univ. Lethbridge (Canada); NAOC (China); CEA, LAM (France); IFSI, Univ. Padua (Italy); IAC (Spain); Stockholm Observatory (Sweden); Imperial College London, RAL, UCL-MSSL, UKATC, Univ. Sussex (UK); and Caltech, JPL, NHSC, Univ. Colorado (USA). This development has been supported by national funding agencies: CSA (Canada); NAOC (China); CEA, CNES, CNRS (France); ASI (Italy); MCINN (Spain); SNSB (Sweden); STFC (UK); and NASA (USA). PACS has been developed by a consortium of institutes led by MPE (Germany) and including UVIE (Austria); KU Leuven, CSL, IMEC (Belgium); CEA, LAM (France); MPIA (Germany); INAF-IFSI/OAA/OAP/OAT, LENS, SISSA (Italy); IAC (Spain). This development has been supported by the funding agencies BMVIT (Austria), ESAPRODEX (Belgium), CEA/CNES (France), DLR (Germany), ASI/INAF (Italy), and CICYT/MCYT (Spain). HIPE is a joint development by the Herschel Science Ground Segment Consortium, consisting of ESA, the NASA Herschel Science Center, and the HIFI, PACS and SPIRE consortia. This work is also based, in part, on observations made with the Spitzer Space Telescope, which is operated by the Jet Propulsion Laboratory, California Institute of Technology under a contract with NASA.

\section{References}

Battersby, C., Bally, J., Jackson, J. M., et al. 2010, ApJ, 721, 222

Benjamin, R., Churchwell, E., Babler, B. L., et al. 2003, PASP, 113, 953

Black, J. H. 1994, in The First Symposium on the Infrared Cirrus and Diffuse Interstellar Clouds, ASP Conf. Ser., 58, 355

Carey, S., Clark, F., Egan, M., et al. 1998, ApJ, 508, 721

Egan, M., Shipman, R., Price, S., et al. 1998, ApJ, 494, 199

Evans, II, N. J., Rawlings, J. M. C., Shirley, Y. L., \& Mundy, L. G. 2001, ApJ, 557,193

Griffin, M. J., Abergel, A., Ade, P., et al. 2010, A\&A, 518, L3

Habing, H. 1968, Bull. Astron. Inst. Neth., 19, 421

Henning, T., Michel, B., \& Stognienko, R. 1995, Planet Space Sci., 43, 1333
Hildebrand, R. 1983, QJRAS, 24, 267

Indebetouw, R., Mathis, J. S., Babler, B. L., et al. 2005, ApJ, 619, 931

Kirk, J., Ward-Thompson, D., \& Andre, P. 2005, MNRAS, 360, 1506

Kirk, J. M., Polehampton, E., Anderson, L. D., et al. 2010, A\&A, 518, L82

Markwardt, C. 2008, in Astronomical Data Analysis Software and Systems

XVIII, ed. D. Bohlender, P. Dowler, \& D. Durand, ASP Conf. Ser., 411, 251

Molinari, S., Swinyard, B., Bally, J., et al. 2010a, A\&A, 518, L100

Molinari, S., Swinyard, B., Bally, J., et al. 2010b, PASP, 122, 314

Nutter, D., Kirk, J., Stamatellos, D., \& Ward Thompson, D. 2008, MNRAS, 384, 775

Ossenkopf, V., \& Henning, T. 1994, A\&A, 291, 943

Ott, S. 2010, in Astronomical Data Analysis Software and Systems XIX, ed. Y. Mizumoto, K.-I. Morita, \& M. Ohishi, ASP Conf. Ser.

Parsons, H., Thompson, M. A., \& Chrysostomou, A. 2009, MNRAS, 399, 1506

Perault, M., Omont, A., Simon, G., et al. 1996, A\&A, 315, 165

Peretto, N., \& Fuller, G. A. 2009, A\&A, 505, 405 (PF09)

Peretto, N., Fuller, G. A., Plume, R., et al. 2010, A\&A, 518, L98

Pilbratt, G. L., Riedinger, J. R., Passvogel, T., et al. 2010, A\&A, 518, L1

Poglitsch, A., Waelkens, C., Geis, N., et al. 2010, A\&A, 518, L2

Preibisch, T., Ossenkopf, V., Yorke, H. W., \& Henning, T. 1993, A\&A, 279, 577

Rathborne, J., Jackson, J., \& Simon, R. 2006, ApJ, 641, 389

Simon, R., Jackson, J., Rathborne, J., \& Chambers, E. 2006a, ApJ, 639, 227 (S06)

Simon, R., Rathborne, J., Shah, R., Jackson, J., \& Chambers, E. 2006b, ApJ, 653,1325

Stamatellos, D., \& Whitworth, A. 2003, A\&A, 407, 941

Stamatellos, D., \& Whitworth, A. 2005, A\&A, 439, 159

Stamatellos, D., Whitworth, A., André, P., \& Ward Thompson, D. 2004, A\&A, 420, 1009

Stamatellos, D., Griffin, M., Kirk, J., et al. 2010, MNRAS, 1204

Teyssier, D., Hennebelle, P., \& Perault, M. 2002, A\&A, 382, 624

Ward-Thompson, D., Kirk, J. M., André, P., et al. 2010, A\&A, 518, L92

Werner, M., Roellig, T., Low, F., et al. 2004, ApJS, 154, 25

Williams, R. J. R., Ward Thompson, D., \& Whitworth, A. P. 2001, MNRAS, 327, 788 